MATHEMATICS OF COMPUTATION

Volume 73, Number 247, Pages 1559-1564

S $0025-5718(03) 01575-8$

Article electronically published on July 1, 2003

\title{
BIQUADRATIC RECIPROCITY AND A LUCASIAN PRIMALITY TEST
}

\author{
PEDRO BERRIZBEITIA AND T. G. BERRY
}

\begin{abstract}
Let $\left\{s_{k}, k \geq 0\right\}$ be the sequence defined from a given initial value, the seed, $s_{0}$, by the recurrence $s_{k+1}=s_{k}^{2}-2, k \geq 0$. Then, for a suitable seed $s_{0}$, the number $M_{h, n}=h \cdot 2^{n}-1$ (where $h<2^{n}$ is odd) is prime iff $s_{n-2} \equiv 0 \bmod M_{h, n}$. In general $s_{0}$ depends both on $h$ and on $n$. We describe a slight modification of this test which determines primality of numbers $h \cdot 2^{n} \pm 1$ with a seed which depends only on $h$, provided $h \not \equiv 0 \bmod 5$. In particular, when $h=4^{m}-1, m$ odd, we have a test with a single seed depending only on $h$, in contrast with the unmodified test, which, as proved by W. Bosma in Explicit primality criteria for $h \cdot 2^{k} \pm 1$, Math. Comp. 61 (1993), 97-109, needs infinitely many seeds. The proof of validity uses biquadratic reciprocity.
\end{abstract}

The Lucasian sequence with seed $s_{0}$ is the sequence $\left\{s_{k}\right\}$ defined from the given initial value $s_{0}$ by the recurrence $s_{k+1}=s_{k}^{2}-2, k \geq 0$. A Lucasian primality test is a primality test involving a Lucasian sequence. The terminology comes from the Lucas-Lehmer test for Mersenne primes (see 4] for historical details):

Theorem 1 (Lucas-Lehmer). Let $p$ be an odd prime, and let $M_{p}=2^{p}-1$ be the corresponding Mersenne number. Let $\left\{s_{k}\right\}$ be the Lucasian sequence with seed 4 . Then $M_{p}$ is prime iff $s_{p-2} \equiv 0 \bmod M_{p}$.

Let $n, h \in \mathbb{N}$ with $h$ odd, $h<2^{n}$, and let $M_{n, h}=h \cdot 2^{n}-1$. The Lucas-Lehmer test generalizes to a Lucasian primality test for $M=M_{h, n}$ as follows:

Theorem 2. Suppose $n \geq 2$. Let $d \in \mathbb{Z}$ satisfy $\left(\frac{d}{M}\right)=-1$, where $(\dot{\bar{M}})$ is the Jacobi symbol. Let $K=\mathbb{Q}(\sqrt{d})$, and let $\mathcal{O}_{K}$ be the ring of integers of $K$. Let $\alpha \in \mathcal{O}_{K}$ satisfy $\left(\frac{\alpha \bar{\alpha}}{M}\right)=-1$, where $\bar{\alpha}$ denotes the conjugate of $\alpha$ in $K$. Then the following are equivalent:

(1) $M$ is prime.

(2) $(\alpha / \bar{\alpha})^{(M+1) / 2} \equiv-1 \bmod M$.

(3) $s_{n-2} \equiv 0 \bmod M$, where $s_{k}$ is the Lucasian sequence with seed $s_{0}=(\alpha / \bar{\alpha})^{h}+$ $(\bar{\alpha} / \alpha)^{h}=\operatorname{Tr}_{K / \mathbb{Q}}(\alpha / \bar{\alpha})^{h}$.

For a proof see [3]. The Lucas-Lehmer test is the special case $d=3, \alpha=$ $-1+\sqrt{3}$ of this theorem. For then $\operatorname{Tr}_{\mathbb{Q}}(\sqrt{3}) / \mathbb{Q}(\alpha)=-4$ whence (the sign being clearly irrelevant) $s_{0}=4$. The generalization differs from the original Lucas-Lehmer test in two respects. First, in the generalized test the seed is a rational number, which may not be an integer; this is not a serious difficulty, since, by inverting the denominator $\bmod M$, one can replace the rational seed by an integer seed. Second,

Received by the editor May 3, 2002 and, in revised form, January 10, 2003.

2000 Mathematics Subject Classification. Primary 11A51, 11 Y11.

(C)2003 American Mathematical Society 
in the generalized test, the seed $s_{0}$ depends on $n$ as well as $h$, while in the LucasLehmer test the seed 4 works for all odd $p$. It is usually easy to find a seed by trial and error. However, following the analogy with Mersenne numbers, our philosophy is to fix $h$ and search for primes in the family $M_{h, n}$ with $n$ increasing. Thus it is certainly desirable to have a seed independent of $n$, if possible. We will have a seed independent of $n$ if we can solve the following problem:

For given $h$, find $d \in \mathbb{Z}, \alpha \in \mathcal{O}_{K}$ where $K=\mathbb{Q}(\sqrt{d})$, such that

$$
\forall n,\left(\frac{d}{M_{h, n}}\right) \neq 1 ;\left(\frac{\alpha \bar{\alpha}}{M_{h, n}}\right) \neq 1
$$

All the above considerations apply also, mutatis mutandis, to numbers of the form $M_{h, n}^{+}=h \cdot 2^{n}+1$, when the primality test in question is Proth's generalization of Pépin's test for Fermat numbers: let $M^{+}=M_{h, n}^{+}$, and let $d$ be an integer such that $\left(\frac{d}{M^{+}}\right)=-1$. Then $M^{+}$is prime iff $d^{\left(M^{+}-1\right) / 2} \equiv-1 \bmod M^{+}$. The problem in this case is to find $d$, depending only on $h$, such that $\forall n,\left(\frac{d}{M_{h, n}^{+}}\right) \neq 1$.

If $n \geq 3, h \not \equiv 0 \bmod 3$, then it is easy to see that, for the generalized Mersenne numbers $M_{h, n}$, as for Mersenne numbers, $d=3, \alpha=-1+\sqrt{3}$ solves the problem; for the $M_{h, n}^{+}$the corresponding problem is solved by $d=3$. The case $h \equiv 0 \bmod 3$ is studied in [1] and [3]. In [3] tables of seeds are given for $M_{h, n}$. In [1] for each $h \equiv 0 \bmod 3, h<10^{5}$, but $h$ not of the form $4^{m}-1$, Bosma exhibits a finite set of pairs $\left(d_{k}, \alpha_{k}\right), d_{k} \in \mathbb{Z}, \alpha_{k} \in \mathbb{Q}\left(\sqrt{d_{k}}\right)$, such that, for any $n$, one of the pairs solves the problem for $M_{h, n}$. On the other hand, for $h$ of the form $4^{m}-1$ he proves there is no such finite set of pairs. Similar results are obtained for the $M_{h, n}^{+}$.

We shall show that, despite Bosma's results, a small modification of the algorithm of Theorem 2 allows us, for fixed $h \not \equiv 0 \bmod 5$, to test primality of $M_{h, n}$ and $M_{h, n}^{+}$ by means of a Lucasian sequence with a seed independent of $n$. In particular when $h=4^{m}-1, m$ odd, we have a single seed.

For any odd integer $k$ we set $k^{*}=\left(\frac{-1}{k}\right) k$. This notation allows us to treat the cases $h \cdot 2^{n} \pm 1$ simultaneously. Note that, if $M=h \cdot 2^{n} \pm 1$, then $M^{*}=( \pm h) 2^{n}+1$. We shall prove:

Theorem 3. Let $M=M_{h, n}=h \cdot 2^{n} \pm 1$, where $h<2^{n-2}-1$ is odd, $h \not \equiv 0 \bmod 5$, and $n \geq 3$. Let $\alpha=-1+2 i \in \mathbb{Z}[i]$ and let $\left\{s_{k}\right\}$ be the Lucasian sequence with seed $s_{0}=(\alpha / \bar{\alpha})^{h}+(\bar{\alpha} / \alpha)^{h}$. Then $M$ is prime iff

- either $M^{*} \equiv \pm 2 \bmod 5$ and $s_{n-2} \equiv 0 \bmod M$

- or $M^{*} \equiv-1 \bmod 5$ and $s_{n-3} \equiv 0 \bmod M$.

The proof of the theorem uses the biquadratic power residue symbol, whose properties we summarize in the following section. Details can be found in [2], Chapter 9.

Biquadratic reciprocity. Let $K=\mathbb{Q}[i]$, and let $R=\mathbb{Z}[i]$ be the ring of integers. Recall that a rational prime $q$ splits in $R$ iff $q \equiv 1 \bmod 4$. Let $\mathfrak{p}$ be a prime ideal of $R$ lying over an odd rational prime, and let $\beta \in R$. The biquadratic residue symbol $\left(\frac{\beta}{\mathfrak{p}}\right)_{4}$ is defined by:

(1) If $\beta \in \mathfrak{p}$, then $\left(\frac{\beta}{\mathfrak{p}}\right)_{4}=0$. 
(2) If $\beta \notin \mathfrak{p}$, then $\left(\frac{\beta}{\mathfrak{p}}\right)_{4}=\omega$, where $\omega$ is the unique fourth root of 1 in $K$ such that

$$
\beta^{\frac{\mathrm{Nm} \mathfrak{p}-1}{4}} \equiv \omega \bmod \mathfrak{p}
$$

where $\mathrm{Nm} \mathfrak{p}$ is the norm of the ideal $\mathfrak{p}$.

(3) If $J \in R$ is an arbitrary ideal and $J=\prod \mathfrak{p}_{i}^{n_{i}}$ is its factorization as a product of prime ideals, then

$$
\left(\frac{\beta}{J}\right)_{4}=\prod\left(\frac{\beta}{\mathfrak{p}_{i}}\right)_{4}^{n_{i}}
$$

Since $R$ is a principal ideal domain, $\mathfrak{p}=(\pi)$ for some irreducible $\pi \in R$, and we normally write $\left(\frac{\beta}{\pi}\right)_{4}$ instead of $\left(\frac{\beta}{\mathfrak{p}}\right)_{4}$. By its very definition the symbol when written in this form depends only on the ideal generated by $\pi$.

We note the following properties:

$$
\begin{aligned}
\left(\frac{\beta \gamma}{\pi}\right)_{4} & =\left(\frac{\beta}{\pi}\right)_{4}\left(\frac{\gamma}{\pi}\right)_{4}, \\
\left(\frac{\beta}{\pi \eta}\right)_{4} & =\left(\frac{\beta}{\pi}\right)_{4}\left(\frac{\beta}{\eta}\right)_{4} \\
\left(\frac{\beta}{\pi}\right)_{4} & =\left(\frac{\bar{\beta}}{\bar{\pi}}\right)_{4} \\
\left(\frac{\beta / \gamma}{\pi}\right)_{4} & =\left(\frac{\beta}{\pi}\right)_{4}\left(\frac{\gamma}{\pi}\right)_{4}^{-1} .
\end{aligned}
$$

We do not need biquadratic reciprocity in full generality, but rather the following proposition (from which the general law can in fact be deduced). An element $\pi \in R$ is primary if $\pi \equiv 1 \bmod (1+i)^{3}$.

Proposition 4. Let $q$ be an odd rational prime and let $\pi \in R, \pi \notin \mathbb{Z}$, be irreducible and primary. Then

$$
\left(\frac{q^{*}}{\pi}\right)_{4}=\left(\frac{\pi}{q}\right)_{4}
$$

For a proof, see Propositions 9.9.6 and 9.9.7 of [2]. We use this result in the following form.

Corollary 5. With the hypotheses of Proposition 4

$$
\left(\frac{q^{*}}{\pi}\right)_{4} \equiv(\pi / \bar{\pi})^{\frac{q^{*}-1}{4}} \bmod q
$$


Proof. Suppose $q \equiv 3 \bmod 4$ so that $q^{*}=-q$ and $q$ is irreducible in $R$. Then

$$
\begin{aligned}
\left(\frac{q^{*}}{\pi}\right)_{4} & =\left(\frac{\pi}{q}\right)_{4} \quad(\text { Proposition 4 }), \\
\left(\frac{\pi}{q}\right)_{4} & \equiv \pi^{\frac{q^{2}-1}{4}} \bmod q\left(\text { definition of the biquadratic symbol, and using } \operatorname{Nm} q=q^{2}\right) \\
& \equiv\left(\pi^{q-1}\right)^{\frac{q+1}{4}} \bmod q \\
& \equiv(\bar{\pi} / \pi)^{\frac{q+1}{4}} \bmod q\left(\text { since } \pi^{q} \equiv \bar{\pi} \bmod q, \text { as is easily seen }\right) \\
& \equiv(\pi / \bar{\pi})^{\frac{q^{*}-1}{4}} \bmod q\left(\text { since } q^{*}=-q\right) .
\end{aligned}
$$

Suppose now $q \equiv 1 \bmod 4$. Then $q^{*}=q$ and $q$ splits in $R$, say $q=\lambda \bar{\lambda}$. We have

$$
\begin{aligned}
\left(\frac{q^{*}}{\pi}\right)_{4} & =\left(\frac{\pi}{q}\right)_{4} \text { (Proposition 4) } \\
\left(\frac{\pi}{q}\right)_{4} & =\left(\frac{\pi}{\lambda \bar{\lambda}}\right)_{4} \\
& =\left(\frac{\pi}{\lambda}\right)_{4}\left(\frac{\pi}{\bar{\lambda}}\right)_{4} \\
& =\left(\frac{\pi}{\lambda}\right)_{4} \overline{\left(\frac{\pi}{\lambda}\right)_{4}} \\
& =\left(\frac{\pi}{\lambda}\right)_{4}\left(\frac{\bar{\pi}}{\lambda}\right)_{4}^{-1} \\
& =\left(\frac{\pi / \bar{\pi}}{\lambda}\right)_{4} \\
& \equiv(\pi / \bar{\pi})^{\frac{q-1}{4}} \bmod q \text { since } \operatorname{Nm}(\lambda)=q
\end{aligned}
$$

which is the desired result, since $q^{*}=q$.

Proof of Theorem 3. The underlying reason for the appearance of Lucasian sequences is the following easily verified proposition.

Proposition 6. Let $\tau$ be an element of norm 1 in a quadratic extension $K$ of $\mathbb{Q}$

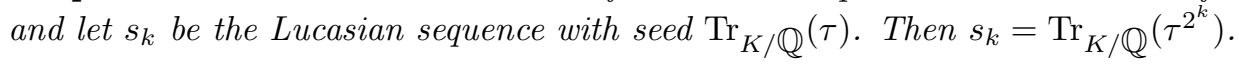

We shall also need the following for the proof of sufficiency of Theorem 3

Lemma 7. Let $K$ be a quadratic extension of $\mathbb{Q}$, let $q$ be an odd rational prime and let $\alpha \in \mathcal{O}_{K}$ be prime to $q$. Set $\tau=\alpha / \bar{\alpha}$. Let $\left\{s_{k}\right\}$ be the Lucas sequence with seed $\operatorname{Tr}(\tau)$. Suppose that, for some $j, s_{j} \equiv 0 \bmod q$. Then $q \equiv \pm 1 \bmod 2^{j+2}$.

Proof. By Proposition [6, $s_{j} \equiv 0 \bmod q$ means $\operatorname{Tr}\left(\tau^{2^{j}}\right) \equiv 0 \bmod q$, i.e., $\tau^{2^{j}}+\bar{\tau}^{2^{j}} \equiv$ 0 mod $q$. Multiplying both sides of the congruence by $\tau^{2^{j}}=\bar{\tau}^{-2^{j}}$ gives

$$
\tau^{2^{j+1}} \equiv-1 \bmod q
$$

Suppose first that $q$ splits in $K$, say $q=\lambda \bar{\lambda}$, where $\lambda$ is an irreducible element of $\mathcal{O}_{K}$ (the ring of integers of $K$ ) with norm $q$. The congruence (5) holds $\bmod \lambda$, which implies that the image of $\tau$ has order $2^{j+2}$ in the group $\left(\mathcal{O}_{K} / \lambda \mathcal{O}_{K}\right)^{*}$. This group has order $\operatorname{Nm} \lambda-1=q-1$, so we conclude $2^{j+2}$ divides $q-1$, i.e., $q \equiv 1 \bmod 2^{j+2}$. 
Suppose $q$ does not split in $K$. From (5) we see that the image of $\tau$ has order $2^{j+2}$ in $\left(\mathcal{O}_{K} / q \mathcal{O}_{K}\right)^{*}$, which is a group of order $q^{2}-1$. But $\tau=\alpha / \bar{\alpha}$ and $\alpha^{q} \equiv \bar{\alpha} \bmod q$, so that $\tau \equiv \alpha^{1-q} \bmod q$, and the image of $\tau$ belongs to the unique subgroup of $\left(\mathcal{O}_{K} / q \mathcal{O}_{K}\right)^{*}$ of order $q+1$. Therefore $2^{j+2}$ divides $q+1$, i.e., $q \equiv-1 \bmod 2^{j+2}$.

From now on, we work in $K=\mathbb{Q}(i)$ and we set $\alpha=-1+2 i$. We note that $\alpha$ is a primary prime in $R=\mathcal{O}_{K}$ and that $2 \equiv-i \bmod \alpha$. Observe also that, since $\alpha \bar{\alpha}=5$, any congruence mod 5 in $R$ implies the same congruence mod $\alpha$.

Now we prove Theorem 3, so that from now on $M$ satisfies the hypotheses of the theorem. We first show that the Lucasian conditions are necessary for primality of $M$. Suppose then that $M$ is prime. Since $n \geq 3$, we have $M \neq 5$, so the hypotheses allow $M^{*} \equiv-1, \pm 2 \bmod 5$, hence $\bmod \alpha$. Suppose first that $M^{*} \equiv \pm 2 \bmod \alpha$. Then $\left(\frac{M^{*}}{\alpha}\right)_{4} \equiv\left(M^{*}\right)^{\frac{\mathrm{Nm} \alpha-1}{4}} \equiv M^{*} \equiv \pm 2 \equiv \mp i \bmod \alpha$. Thus, by definition of the biquadratic symbol, $\left(\frac{M^{*}}{\alpha}\right)_{4}=\mp i$. Applying Corollary 5 ,

$$
(\alpha / \bar{\alpha})^{\frac{M^{*}-1}{4}} \equiv \mp i \bmod M
$$

whence

$$
(\alpha / \bar{\alpha})^{\frac{M^{*}-1}{2}} \equiv-1 \bmod M
$$

Since $\left(M^{*}-1\right) / 2=( \pm h) \cdot 2^{n-1}$, this gives $(\alpha / \bar{\alpha})^{( \pm h) \cdot 2^{n-1}} \equiv-1 \bmod M$. We thus find $\operatorname{Tr}(\alpha / \bar{\alpha})^{( \pm h) \cdot 2^{n-1}}=\operatorname{Tr}(\alpha / \bar{\alpha})^{h \cdot 2^{n-1}} \equiv-2 \bmod M$. By Proposition 6 , this is equivalent to $s_{n-1} \equiv-2 \bmod M$, and $s_{n-2} \equiv 0 \bmod M$ follows from the recurrence satisfied by the $s_{k}$.

Suppose now that $M^{*} \equiv-1 \bmod 5$. Then, direct calculation of the biquadratic symbol, as in the first case, gives $\left(\frac{M^{*}}{\alpha}\right)_{4}=-1$. By Corollary 5 again

$$
(\alpha / \bar{\alpha})^{\frac{M^{*}-1}{4}} \equiv-1 \bmod M
$$

i.e., $(\alpha / \bar{\alpha})^{( \pm h) \cdot 2^{n-2}} \equiv-1 \bmod M$ from which, arguing as in the first case, $s_{n-3} \equiv$ $0 \bmod M$ follows. This completes the proof of necessity.

We now turn to the proof of sufficiency. This uses a standard method, which is, to prove that the hypotheses imply that if $q$ is an arbitrary prime divisor of $M$, then $q>\sqrt{M}$.

With the notation of the theorem assume one or other of the possible congruences on the $s_{k}$ is satisfied. Let $q$ be a prime divisor of $M$, and let $\tau=\alpha / \bar{\alpha}$. Then the hypotheses imply $q \neq 5$ so Lemma 7 applies, and either $q \equiv \pm 1 \bmod 2^{n-1}$ or $q \equiv$ $\pm 1 \bmod 2^{n}$. From these congruences it follows easily that in all cases $q \geq 2^{n-1}-1$, whence $q^{2} \geq 2^{2 n-2}-2^{n}+1$. On the other hand $M=h \cdot 2^{n} \pm 1 \leq h \cdot 2^{n}+1$. But $h<2^{n-2}-1$ by hypothesis, whence $M<2^{2 n-2}-2^{n}+1 \leq q^{2}$, as we wished to prove. 


\section{REFERENCES}

1. Wieb Bosma. Explicit primality criteria for $h \cdot 2^{k} \pm 1$. Math. Comp., 61(203):97-109, S7-S9, 1993. MR 94c:11005

2. K. Ireland and M. Rosen. A classical introduction to modern number theory. Springer Verlag, 1990. MR 92e:11001

3. Hans Riesel. Lucasian criteria for the primality of $N=h \cdot 2^{n}-1$. Math. Comp., 23:869-875, 1969. MR 41:6773

4. Hugh C. Williams. Édouard Lucas and Primality Testing, volume 22 of Canadian Math. Society Series of Advanced Texts. John Wiley and Sons, 1998. MR 2000b:11139

Departamento de Matemáticas Puras y Aplicadas, Universidad Simón Bolívar, CaraCAs, Venezuela

E-mail address: pedrob@usb.ve

Departamento de Matemáticas Puras y Aplicadas, Universidad Simón Bolívar, CaraCAS, Venezuela

E-mail address: berry@usb.ve 